\title{
WHY BEHAVIORISM, TO SURVIVE AND TRIUMPH, MUST ABANDON ITS VERY NAME: AN OPEN LETTER
}

\begin{abstract}
Direct contingency analysis has been shown to be important. Instead of this success providing a powerful foundation for major advances in all of the important areas of psychology, I fear that it is becoming a gold plated cage. My evidence: relatively narrow areas of interest are dominating the field, both applied and basic. We are not expanding, and a field not busy being born is a field that is busy dying. (Hayes, 2001, p. 61)
\end{abstract}

A hallmark virtue of behaviorism and behavior analysis is its adherence to a thoroughgoing empiricism with a commitment to discovering the lawfulness of behavior and the development of procedures for its prediction and control. Ultimately, the aspects of behavior that can become a subject matter for a behavioristic examination are dependent upon the capability of the tools available that can measure those aspects. Thus, respondent and operant behaviors become grist for a behavioristic analysis only because we have the devices (e.g. Skinner box, cumulative record, Pavlov's gastric fistula) available to measure and control for these responses. Unfortunately, if schools of thought become defined through the unique methodology they employ rather than the empirical principles they espouse, then these schools of thought become ultimately built on artificial principles. In the case of behaviorism, the dogmatic reliance on methodological at the expense of empirical principles not only constrains behaviorism, but as I must agree with the aforementioned quotation, may ultimately kill it.

The fact that respondent and operant conditioning have only recently been analyzed using uniform methodological principles (Donahoe \& Palmer, 1993) underscores the fact that different procedures can be wrongly imputed to underscore subject matters that are incommensurable. However, the methodological divisions that can separate practitioners into different camps is presently repeating itself once again, and with results that are of key importance to the future of behaviorism.

Presently, the rapid development of new and powerful experimental tools (MRI, in vivo microdialysis, neuronal modeling, etc.) that can map neural processes has demonstrated that neuromodulator production (Barrett and Hoffman, 1991), micro-cellular processes (Stein et al. 1993), and many other neural events can now be mapped to environmental contingencies. These findings are no less behavioristic than Pavlov's measure of gastric secretions, yet because the experimental methods and devices are so different from those customarily employed by behavior analysts, the tools themselves have defined a subject matter, namely neuropsychology, that seems quite different from behaviorism, even though the former is in principle behavioristic.

A major emerging concept in cognitive neuropsychology that is being examined through the use of operant principles is the concept of embodiment, a notion that has received scant attention from behavior analysts. Embodiment, 
which is now held as a first principle by the major voices in neuropsychology today (e.g. Antonio Damasio (1994), Jaak Panksepp (1999)) in effect holds that behavior is not only instantiated by environmental contingencies, but is continuously guided by somatic events (e.g. muscle tension, neuromodulator production, hormonal responses, etc.) that are instigated by and mediate the effectiveness of those contingencies. Embodiment is different from the more general concept of emotion because unlike the latter, it possesses 'theoretical coherence'. In other words, embodied events represent measurable somatic responses that vary in time and across other independent measures (e.g. response contingencies) that can be altered and replicated.

Embodiment should be particularly of importance to behaviorism for the simple reason that embodiment is now at the root of contemporary bio-behavioral definitions of reinforcement. Current discrepancy models of reward hold that reinforcement is coextensive with the production of the neuromodulator dopamine that fixes attention, assigns appetitive value to perceptual events, and increases synaptic or neural efficiency. The concept of embodiment as reflected in the activity of dopamine neurons represents a particular challenge to the canonical behavioristic representation of a reinforcer as a discrete event that controls or motivates behavior as the consequence of a fixed response contingency. Rather, neuroscientific theories of reinforcement assign reinforcement to the prediction error that derives from an individual's moment-to-moment perception of prevailing response contingencies. That is, reinforcement is a continuous and variable and not an intermittent and fixed event, and is relative to prediction error perceived at any moment in time. This view, which is presently the dominant interpretation of learning in neuroscience proposes (Hollerman and Schultz, 1998) that: "Learning depends on the extent to which behavioral outcomes are different than predicted, being governed by the discrepancy of 'error' between outcome and prediction. Outcomes that affect learning in this way are termed 'reinforcers.'...Learning proceeds when outcomes occur that are not fully predicted, then slows down as outcomes become increasingly predicted and ends when outcomes are fully predicted" (p. 304). Furthermore, "the magnitude of dopamine responses to reward reflect the degree of reward predictability during individual learning episodes..." (p. 304). Although a dopaminergic based discrepancy theory was first ventured by Donahoe's unified reinforcement principle (Donahoe and Palmer, 1993), the fact that dopamine production co-varies with the magnitude and timing of prediction error, and does not just mark prediction error impacts not just the quality of reinforcement, but also the attendant quality of subjective experience. In other words, the phenomenology of happiness, or its subjective feeling, can now be rooted to actual physiological processes that can be simply conceived. These facts can be simply illustrated through three examples that appeal to common experience. 


\section{Mind EXPERIMENTS}

Take a simple piecework task, such as pulling a lever many times a minute to stamp out buttons on an assembly line. The reward for performing this task, namely a salary, is wholly predictable in its timing and its amount. However, if the timing of the reward as well as its size radically varied over time, then although the average weekly salary would remain the same, the worker could be rewarded substantially, marginally, or not at all upon the after each lever pull. The latter example, which makes the button machine into a slot machine, will result in the otherwise tired and bored worker becoming suddenly animated, interested, excited, and ironically, indifferent to the reasonable expectation that he or she may likely have a net loss at the end of the week. The manipulation of prediction error alone therefore transforms an onerous "extrinsically" motivated task into a highly desirable "intrinsically" motivated task.

As another example, consider a non-rote task that requires an individual to figure out a puzzle or other problem. The solution for novel problem solving task also involves unpredictable prediction errors, when progress towards solving the problem occurs intermittently and surprisingly as different options are considered. If the prediction errors are positive, rapid, and high, then we have a lot of dopamine produced and a corresponding enjoyable, flowing, peak, or otherwise pleasurable experience. Similar activities that involve high, rapid, and positive prediction error are creative, sporting, gaming, or other tasks.

As a final example, let us consider the exemplar of all positively motivated individuals: Shakespeare. If the fanciful movie Shakespeare in Love is to be believed, Shakespeare's genius was spurred by a confluence of motivators, including girlfriends, competitors, fellow actors and investors, not to mention the approval of the crowd, the Queen, and posterity. It was indeed a volatile matrix of uncertainty that excited the imagination and the pen, giving us a play, Romeo and Juliet, a sublime mixture of pratfalls and poetry that appealed to crowd and Queen alike. But what indeed motivated Shakespeare, uncertainty or contingency? Simple, remove the uncertainty (but not the contingency) and the edifice collapses, and Shakespeare then knows all the right moves, and would likely become bored to tears. He would be no more inspired than a baseball player who knows the final score beforehand, or a gambler who knows the impending face of each card. Shakespeare in Love would become Shakespeare in Hell, a presumption that has at least literary precedent.

In an episode of the classic TV series The Twilight Zone, a burglar gets shot and killed, and is met by a jovial fellow who introduces him to a world where he can have anything he wants, from women to power to fame. Unfortunately, everything is totally predictable, from the role of a die to a woman's sigh. He protests that everything has become boring and dull, and requests to be shipped off to hell, where at least he could play chess with the devil. The man laughs, and says to the shocked burglar, "Whatever makes you think that you are in heaven?" 


\section{The MARRIAGe OF VIRTUE AND HAPPINESS}

In keeping with these findings, dopamine has recently been demonstrated to scale up or down with changes in the probability and importance of expected events (Breiter, Aharon, Kahneman, Anders, \& Shizgal, 2001) and with the frequency of cognitive set shifting between expected events, such as in creative behavior (Fried et al. 2001) and video game playing (Koepp et al. 1998). Elevated dopamine production also occurs or displays "behavioral momentum" after the conditions or contingencies that elicit it have been altered or withdrawn (Ashby, Isen, \& Turken, 1999). In particular, because dopamine bestows an appetitive value on behavior, it will alter behavior in ways that cannot be predicted as a function of a consideration of perceptual events alone. That is, response contingencies not only provide a perceptual road map whereby we may categorize value, but also continuously elicit and control somatic responses that confound or skew value. For example, if I do a good deed (e.g. hit a base hit for the Diamondbacks in the ninth inning of game 7 in the World Series), I consider the positive and unpredictable ways my audience will react, and if I do a bad deed (be the guy who threw that same pitch), I will consider the negative albeit unpredictable reactions of the same audience. The prediction error is virtual, not real, and reflects as-if relationships between perceptual events. Yet the pain or pleasure associated with that prediction error is quite real, and serves to modulate or change behavior (e.g. whether you sulk in your dugout or cavort in your clubhouse with champagne). Because dopamine maps to as-if prediction errors, it essentially can influence behavior due to virtual as well as real events. The ability to feel another's pain, which is the root of empathy, does not mean that another's pain is actually modeled, but rather that dopamine production is increased or suppressed due to our consideration of the modeled positive or negative responses of other people. This brings us to a very interesting conclusion. If reinforcers are equivalent to arranging positive and unpredictable prediction error, then happiness

if simply finding ways to increase positive prediction error in our daily lives. Yet to increase positive prediction error entails the ability to model other people's responses, and to be effected by knowledge of their pleasure and approval and their disapproval and pain. Thus to be reinforced is to be empathetic, and to increase empathy is ultimately to increase happiness and moral virtue.

\section{The Bugbear of Measurement}

Unfortunately, since neuromodulator activity cannot be as easily charted as say a bar press or key peck, indirect and less precise methods must also be used to account for the somatic events elicited by response contingencies. In particular, these methods entail subjective reports of pain and pleasure and how they map to abstract elements of environmental contingencies. However, as behaviorists are amply aware, the use of subjective reporting suffers from a tendency towards unreliability, and at worse an embrace of mentalism. An exemplar of this problem is the work of the psychologist M. Csikszentmihalyi $(1990,1997)$. After polling 
thousands of individuals in various circumstances of work and play, Csikszentmihalyi demonstrated a high correlation of self-reports of pleasurable or ecstatic states to elements of response contingencies (a "demand/skill" match). Unfortunately, Csikszentmihalyi did not ground his observations to neural events, but rather to mentalistic entities such as "psychic energy", "undreamed states of consciousness" and the like. The behavioral contingencies that may be reliably inferred from Csikszentmihalyi's work are precisely the same as those that may be mapped to dopamine release, yet the thoroughgoing mentalism that characterizes Csikszentmihalyi's work has rendered it opaque to behavior analysis, and hence the behaviorist community has ignored it.

Mentalism is ubiquitous in our lives, as we couldn't communicate with each other otherwise. Yet we manage to live with the mental homunculi of will, desire, courage, consciousness, and other psychological faculties because we can continuously qualify what we hear, feel and see by a deep knowledge of physical reality (e.g., Einsteinian concepts of time, Darwinian evolution, etc.) But just as a medical doctor must listen to the unreliable patient reports of aches and pains as a way to inform more accurate diagnostic and therapeutic procedures, so too must behaviorists be able to use the imprecision of self reports to inform sound behavioral interventions. Behaviorism has been marginalized by an intolerance of any imprecision in exchange for an incomplete accounting for behavior that has in turn limited the effectiveness of its practical procedures. It has allowed great swaths of its rightful subject matter be co-opted by mentalistic models for behavior (e.g. "flow states," intrinsic vs. extrinsic motivation, needs for achievement, etc.), and has suffered itself to be commonly relegated to the ash can of science by many popular and academic pundits on psychology whom one might think would know better. This can only change if behaviorists become, well, more behavioristic, and account more thoroughly for the subject matter (namely behavior in all its covert and overt manifestations) that they purport to explain. But as I will note with more than a little irony, a true thoroughgoing behaviorism makes the very term behaviorism redundant and useless.

\section{SKINNER OUT OF THE BOX}

The focus on embodiment, which is so characteristic of modern neuropsychology, and so reflective of behavioristic principles, has been near totally ignored by contemporary behaviorism. The supreme irony of this statement is that this ignorance runs counter to the inductive principles that were the hallmark of Skinner's perspective on psychological science. As Donahoe and Palmer (1993)

note, "the distinction between observed and unobserved events is not a distinction between kinds of events, but between the resolving power of different technologies" (p. 5) Also, "The view that microbehavioral events are part of a science of behavior was termed radical behaviorism by Skinner. Such unobserved events are admissible so long as they are not accorded characteristics other than those of observed events that have been subjected to experimental analysis" (p. 7). Thus, in Donahoe and Palmer's opinion methodological behaviorism must expand 
to include events that are unobserved at the behavioral level for two reasons: "(A) The distinction between unobserved and observed events is not a fixed line that separates different kinds of events, but a fuzzy boundary that advances as technology progresses. (B) The incorporation of sub behavioral events may improve the precision of the functional relations between events measured at the behavioral level" (p. 7).

Behaviorism has long justified itself by a philosophy that exalts prediction and control over theoretical explanation. However, in order to maximize the predictive power of its procedures, it must now consider all aspects of behavior from self-reports to overt behavior to covert and micro-behavioral responses, and thus ironically back into the explanatory models it once ignored. Unfortunately however, if behaviorism is practically defined through a distinctive data language and methodology, the expansion of a methodological behaviorism to encompass subjective and micro-behavioral indices of behavior broadens that language and methodology. It thus dissolves the linguistic and methodological distinctiveness of behaviorism, and identifies it instead with general inductive and empiricist principles. Thus behaviorism dies, but rises again as the culmination of psychological science.

The fact that behaviorism is not a mere school of psychology, but is psychology, must force behaviorists to expand their vision, if for no other reason than to maintain the integrity of the principles that founded behaviorism to begin with. The current resistance to this necessity has been noted in a recent article by the distinguished neuropsychologist Jaak Panksepp (www.neuro-psa.com/pank. htm.) In this acerbic paper, Panksepp notes with contempt the stubborn reluctance of intellectually 'autistic' psychologists who espouse the empirical virtues of science, but refrain from the barest attempt to use and integrate the empirical methods and data languages of science. Calling to task psychoanalysts, experimental psychologists, and (methodological) behaviorists, Panksepp noted:

In any event, mind, brain, and behavior are completely interdependent-and we cannot understand the resulting integrated processes unless we are willing to take all three perspectives concurrently to the study of animate actions. If behaviorists continue to just study emotional behaviors, with no concern for the affective experience of animals, we will continue to have a dualistic view of the brain. If experimental psychologists and psychoanalysts choose to pursue only the psychic reflections of brain processes, when the obvious three pronged solution is readily available, we shall surely continue with another century of misunderstanding. However, if we come to terms with the full tri-dimensional complexities, accepting that there are various unified psychic states arising from neurodynamic processes that arouse behavioral urges, then we may eventually have unified approaches to knowledge that can truly nourish the intellectual curiosity of future generations of students (p. 13).

Ultimately, there is only one question I will forward. What is behaviorism? Is behaviorism defined by a scientific philosophy, or does it merely reflect the use of a specific type of methodology and the interpretations of the data produced by that 
method? If it embodies science, then let it embrace science, and integrate itself at long last with those other approaches that see beauty, feel pain, know the molecules that make our behavior and passions, and of course, the overt responses that we know as behavior.

\section{A. J. Marr (lanna@bellsouth.net) drmezmer.com \\ Benecom Technologies, Inc. New Orleans, La.}

There are three major sources of information on unified reinforcement or discrepancy theory, and one that is a bit odd. The first represents the work of the bio-behaviorists John Donahoe and David Palmer. Their Unified Principle of Reinforcement is the first systematic presentation of a discrepancy theory of reinforcement, and scholarly articles on their work can be found on the web site of the Journal of the Experimental Analysis of Behavior (JEAB).

The most lucid and up to date accounts of discrepancy theory are also found on the web. The Google web directory contains quite a few PDF files on articles by the neuroscientist Wolfram Schultz that are lucid yet rigorously argued. Schultz is presently the major figure in neuroscience who has comprehensively examined the neuropsychology of reward.

The third major source of information on unified reinforcement theory is found in the 1998 book, 'Affective Neuroscience', by Jaak Panksepp. Panksepp is also well represented in the web. His comment (from chapter 8 of his book) is instructive as to the untenability of the concept "extrinsic reward."

From the behaviorist perspective, the incentive properties of a reward were traditionally defined in terms of attributes such as the quality, quantity, and delay of reward rather than in terms of any conception of what the nervous system experiences and undergoes when it is confronted by highly desirable objects. In fact, the high incentive state, from the nervous system perspective, may be the arousal of an emotive process that invigorates search and foraging behaviors. In other words, the unconditional incentive state within the brain may largely consist of the arousal of a psycho-behavioral integrative system (e.g., seeking) of the brain. An increased number of studies measuring DA (dopaminergic) cellular activity, as well as dopamine release in the pathways emanating from the VTA (e.g. a midbrain structure-my note), now indicate that this system is highly tuned to stimuli that predict rewards, rather than to rewards themselves."

Finally, if you choose to pursue my babbling argument a bit further, I have included five articles on biobehaviorism and its relevance to the positive psychology, the intrinsic/extrinsic motivation controversy, the flow response, and other related topics on my web site drmezmer.com. This site will no doubt be jarring to the casual observer more familiar with staid academic websites. Just skip 
the other topics, and go to "flow, a reverent explanation," and you will find these articles, and enter the happy land of journalese.

\section{REFERENCES:}

Ashby, F. G., Isen, A. M., \& Turken U. (1999). A neuropsychological theory of positive affect and its influence on cognition. Psychological Review, 106 (3), 529-550.

Barrett, J. E. \& Hoffman, S. M. (1991) Neurochemical changes correlated with behavior maintained under fixed-interval and fixed-ratio schedules of reinforcement. Journal of the Experimental Analysis of Behavior, 56, 395-405.

Breiter, H. C., Aharon, I., Kahneman, D., Anders, D., \& Shizgal, P. (2001) Functional imaging of neural responses to expectancy and experience of monetary gains and losses, Neuron, 30, 619-639.

Csikszentmihalyi, M. (1990). Flow, the psychology of optimal experience. New York: Harper Collins.

Csikszentmihalyi, M. (1997). Finding flow. New York: Basic Books.

Damasio, A. (1994). Descartes' error: Emotion, reason and the human brain. New York: Avon Books.

Donahoe, J. W. \& Palmer, D. C. (1993). Learning and complex behavior. Needham Heights, Ma.: Allyn and Bacon.

Fried, I., Wilson, C. L, Morrow, J. W., Cameron, K. A., Behnke, E. D., Ackerson, L. C. \& Maidment, N. T. (2001) Increased dopamine release in the human amygdala during performance of cognitive tasks. Nature Neuroscience, 4(2), 201-206.

Hayes, S. C. (2001) The greatest dangers facing behavior analysis today. The Behavior Analyst Today 2(2), 61-63.

Hollerman, J. R., \& Schultz, W. (1998) Dopamine neurons report an error in the temporal prediction of reward during learning. Nature Neuroscience, 1(4): 304-309.

Koepp, M. J., Gunn, R. N., Lawrence, A. D., Cunningham, V. J., Dagher, A., Jones, T., Brooks, D. J. Bench C. J., \& Grasby, P. M. (1998). Evidence for striatal dopamine release during a video game. Nature, 393, 266-268.

Lakoff, G., \& Johnson, M. (1999). Philosophy in the flesh: The embodied mind and its challenge to Western thought, New York: Basic Books.

Panksepp, J. (1998). Affective neuroscience. New York: Oxford University Press.

Schultz, W. (1998). Predictive reward signal of dopamine neurons. Journal of Neurophysiology, 80(1) 1-27.

Stein, L., Xue, B. G. \& Belluzzi, J. D. (1993). A cellular analogue of operant conditioning. Journal of the Experimental Analysis of Behavior, 60, 41-53. 